\title{
ALGEBRAIC SYSTEMS WITH LIPSCHITZ PERTURBATIONS
}

\author{
GIOVANNI MOLICA BISCI AND DUŠAN REPOVŠ
}

\begin{abstract}
By using variational methods, the existence of infinitely many solutions for a nonlinear algebraic system with a parameter is established in presence of a perturbed Lipschitz term. Our goal was achieved requiring an appropriate behavior of the nonlinear term $f$, either at zero or at infinity, without symmetry conditions.
\end{abstract}

\section{INTRODUCTION}

In many cases a problem in a continuous framework can be handled by using a suitable method from discrete mathematics, and conversely. For instance, let us consider the following relations

$$
\begin{gathered}
{[u(i+1, j)-2 u(i, j)+u(i-1, j)]+[u(i, j+1)-2 u(i, j)+u(i, j-1)]} \\
+\lambda f((i, j), u(i, j))=0, \quad \forall(i, j) \in \mathbb{Z}[1, m] \times \mathbb{Z}[1, n],
\end{gathered}
$$

under the Dirichlet boundary conditions

$$
\begin{aligned}
u(i, 0) & =u(i, n+1)=0, \quad \forall i \in \mathbb{Z}[1, m], \\
u(0, j)=u(m+1, j)=0, & \forall j \in \mathbb{Z}[1, n],
\end{aligned}
$$

where $f: \mathbb{Z}[1, m] \times \mathbb{Z}[1, n] \times \mathbb{R} \rightarrow \mathbb{R}$ denotes a continuous function and $\lambda$ is a positive parameter. As pointed out by Galewski and Orpel in [5], the above problem serves as the discrete counterpart of the following continuous one:

$$
\left\{\begin{array}{l}
\frac{\partial^{2} u}{\partial x^{2}}+\frac{\partial^{2} u}{\partial y^{2}}+\lambda f((x, y), u(x, y))=0 \\
u(x, 0)=u(x, n+1)=0, \quad \forall x \in(0, m+1) \\
u(0, y)=u(m+1, y)=0, \quad \forall y \in(0, n+1)
\end{array}\right.
$$

However, the results obtained here (see Theorem 1 below) cannot be directly achieved by proving the existence of solutions for the above equation. The modeling/simulation of certain nonlinear problems from economics, biological neural networks, optimal control and others, enforced in a natural manner a rapid development of the theory of discrete equations (see for instance [26] and references therein).

2010 Mathematics Subject Classification. Primary: 39A10; Secondary: 47J30, 58E05.

Key words and phrases. Discrete nonlinear boundary value problems; infinitely many solutions; difference equations, critical points theory.

Received 09/10/2014, accepted 07/02/15.

The manuscript was realized within the auspices of the INdAM - GNAMPA Project 2014 titled: Propriet geometriche ed analitiche per problemi non-locali and the SRA grants P1-0292-0101 and J1-5435-0101. 
In this paper, motivated by this increasing interest, we study the following algebraic system

$$
A u=\lambda f(u)+h(u)
$$

in which $u=\left(u_{1}, \ldots, u_{n}\right)^{t} \in \mathbb{R}^{n}$ is a column vector, $A=\left(a_{i j}\right)_{n \times n}$ is a positive-definite matrix, $f(u):=\left(f_{1}\left(u_{1}\right), \ldots, f_{n}\left(u_{n}\right)\right)^{t}$, where the functions $f_{k}: \mathbb{R} \rightarrow \mathbb{R}$ are assumed to be continuous for every $k \in \mathbb{Z}[1, n]:=\{1,2, \ldots, n\}$, and $\lambda$ is a positive parameter.

Moreover,

$$
h(u):=\left(h_{1}\left(u_{1}\right), \ldots, h_{n}\left(u_{n}\right)\right)^{t},
$$

where, for every $k \in \mathbb{Z}[1, n]$, the functions $h_{k}: \mathbb{R} \rightarrow \mathbb{R}$ are Lipschitz continuous with constants $L_{k} \geq 0$, that is:

$$
\left|h_{k}\left(t_{1}\right)-h_{k}\left(t_{2}\right)\right| \leq L_{k}\left|t_{1}-t_{2}\right|,
$$

for every $t_{1}, t_{2} \in \mathbb{R}$, and $h_{k}(0)=0$.

A large number of discrete problems can be formulated as special cases of the nonperturbed $(h=0)$ algebraic system, namely $\left(S_{A, \lambda}^{f}\right)$; see, for instance, the papers [23, 25, 26, $27,28]$ and references therein. We also point out that the case

$$
A:=\left(\begin{array}{ccccc}
2 & -1 & 0 & \ldots & 0 \\
-1 & 2 & -1 & \ldots & 0 \\
& & \ddots & & \\
0 & \ldots & -1 & 2 & -1 \\
0 & \cdots & 0 & -1 & 2
\end{array}\right)_{n \times n}
$$

has been considered in order to study the existence of nontrivial solutions of nonlinear second-order difference equations $[12,13,15]$. Moreover, as it is well-known, boundary value problems involving fourth-order difference equations such as

$\left(D_{\lambda}^{f}\right)$

$$
\left\{\begin{array}{l}
\Delta^{4} u_{k-2}=\lambda f_{k}\left(u_{k}\right), \quad \forall k \in \mathbb{Z}[1, n] \\
u_{-2}=u_{-1}=u_{0}=0 \\
u_{n+1}=u_{n+2}=u_{n+3}=0,
\end{array}\right.
$$

can also be expressed as the problem $\left(S_{A, \lambda}^{f}\right)$, where $A$ is the real symmetric and positive definite matrix of the form

$$
A:=\left(\begin{array}{ccccccccc}
6 & -4 & 1 & 0 & \cdots & 0 & 0 & 0 & 0 \\
-4 & 6 & -4 & 1 & \cdots & 0 & 0 & 0 & 0 \\
1 & -4 & 6 & -4 & \cdots & 0 & 0 & 0 & 0 \\
0 & 1 & -4 & 6 & \cdots & 0 & 0 & 0 & 0 \\
& & & & \ddots & & & & \\
0 & 0 & 0 & 0 & \cdots & 6 & -4 & 1 & 0 \\
0 & 0 & 0 & 0 & \cdots & -4 & 6 & -4 & 1 \\
0 & 0 & 0 & 0 & \cdots & 1 & -4 & 6 & -4 \\
0 & 0 & 0 & 0 & \cdots & 0 & 1 & -4 & 6
\end{array}\right) .
$$

Further, general references on difference equations and their applications can be found e.g. in $[1,10]$.

Here, by using variational methods, under the key assumption that

$$
L:=\max _{k \in \mathbb{Z}[1, n]} L_{k}<\lambda_{1},
$$


where $\lambda_{1}$ is the first eigenvalue of the matrix $A$, we determine open intervals of positive parameters such that problem $\left(S_{A, \lambda}^{f, h}\right)$ admits either an unbounded sequence of solutions, provided that the nonlinearity $f$ has a suitable behaviour at infinity (Theorem 3 ), or a sequence of pairwise distinct solutions that converges to zero, if a similar behaviour occurs at zero (see Theorem 4).

Our main tool is a recent critical point result obtained by Ricceri and recalled here in a convenient form (see Theorem 2).

A special case of our results reads as follows (see Remark 4).

Theorem 1. Let $z: \mathbb{R} \rightarrow \mathbb{R}$ be a nonnegative and continuous function. Assume that

$$
\liminf _{t \rightarrow+\infty} \frac{\int_{0}^{t} z(\xi) d \xi}{t^{2}}=0, \quad \limsup _{t \rightarrow+\infty} \frac{\int_{0}^{t} z(\xi) d \xi}{t^{2}}=+\infty .
$$

Then, for each $\lambda>0$, and for every Lipschitz continuous function $h: \mathbb{R} \rightarrow \mathbb{R}$ with Lipschitz constant $L_{h}<\lambda_{A}$ (where $\lambda_{A}$ is the first eigenvalue of the matrix A defined in Section 4), the following discrete problem

$$
\begin{gathered}
{[u(i+1, j)-2 u(i, j)+u(i-1, j)]+[u(i, j+1)-2 u(i, j)+u(i, j-1)]} \\
+\lambda z(u(i, j))+h(u(i, j))=0, \quad \forall(i, j) \in \mathbb{Z}[1, m] \times \mathbb{Z}[1, n]
\end{gathered}
$$

with boundary conditions

$$
\begin{aligned}
& u(i, 0)=u(i, n+1)=0, \quad \forall i \in \mathbb{Z}[1, m], \\
& u(0, j)=u(m+1, j)=0, \quad \forall j \in \mathbb{Z}[1, n],
\end{aligned}
$$

admits an unbounded sequence of solutions.

Finally, for completeness, we just mention here that there is a vast literature on nonlinear difference equations based on fixed point and upper and lower solution methods (see $[2,8]$ ). For related topics see the works $[3,6,7,22]$. For a complete and exhaustive overview on variational methods we refer the reader to the monographs $[11,20]$.

\section{Abstract Setting}

Let $(X,\|\cdot\|)$ be a finite-dimensional Banach space and let $J_{\lambda}: X \rightarrow \mathbb{R}$ be a function satisfying the following structure hypothesis:

$(\Lambda)$ for all $u \in X, J_{\lambda}(u):=\Phi(u)-\lambda \Psi(u)$ where $\Phi, \Psi: X \rightarrow \mathbb{R}$ are two functions of class $C^{1}$ on $X$ with $\Phi$ coercive, i.e. $\lim _{\|u\| \rightarrow \infty} \Phi(u)=+\infty$, and $\lambda$ is a real positive parameter.

Moreover, provided that $r>\inf _{X} \Phi$, put

$$
\varphi(r):=\inf _{u \in \Phi^{-1}(]-\infty, r[)} \frac{\left(\sup _{v \in \Phi^{-1}(]-\infty, r[)} \Psi(v)\right)-\Psi(u)}{r-\Phi(u)},
$$

and

$$
\gamma:=\liminf _{r \rightarrow+\infty} \varphi(r), \quad \delta:=\liminf _{r \rightarrow\left(\inf _{X} \Phi\right)^{+}} \varphi(r) .
$$

Clearly, $\gamma \geq 0$ and $\delta \geq 0$. When $\gamma=0$ (or $\delta=0$ ), in the sequel, we agree to read $1 / \gamma$ (or $1 / \delta)$ as $+\infty$. 
Theorem 2. Assuming that the condition $(\Lambda)$ holds, one has

(a) If $\gamma<+\infty$ then, for each $\lambda \in] 0,1 / \gamma[$, the following alternative holds: either

$\left(\mathrm{a}_{1}\right) J_{\lambda}$ possesses a global minimum,

or

$\left(\mathrm{a}_{2}\right)$ there is a sequence $\left\{u_{m}\right\}$ of critical points (local minima) of $J_{\lambda}$ such that $\lim _{m \rightarrow \infty} \Phi\left(u_{m}\right)=+\infty$.

(b) If $\delta<+\infty$ then, for each $\lambda \in] 0,1 / \delta[$, the following alternative holds: either

$\left(\mathrm{b}_{1}\right)$ there is a global minimum of $\Phi$ which is a local minimum of $J_{\lambda}$, or

$\left(\mathrm{b}_{2}\right)$ there is a sequence $\left\{u_{m}\right\}$ of pairwise distinct critical points (local minima) of $J_{\lambda}$, with $\lim _{m \rightarrow \infty} \Phi\left(u_{m}\right)=\inf _{X} \Phi$, which converges to a global minimum of $\Phi$.

Remark 1. Theorem 2 is the finite-dimensional version of the quoted multiplicity result of Ricceri from [21].

As ambient space $X$, consider the $n$-dimensional Banach space $\mathbb{R}^{n}$ endowed by the norm

$$
\|u\|:=\left(\sum_{k=1}^{n} u_{k}^{2}\right)^{1 / 2} .
$$

Set $\mathfrak{X}_{n}$ be the class of all symmetric and positive-definite matrices of order $n$. Further, we denote by $\lambda_{1}, \ldots, \lambda_{n}$ the eigenvalues of $A$, ordered as follows $0<\lambda_{1} \leq \ldots \leq \lambda_{n}$.

It is well-known that if $A \in \mathfrak{X}_{n}$, then for every $u \in X$, one has

$$
\lambda_{1}\|u\|^{2} \leq u^{t} A u \leq \lambda_{n}\|u\|^{2}
$$

and

$$
\|u\|_{\infty} \leq \frac{1}{\sqrt{\lambda_{1}}}\left(u^{t} A u\right)^{1 / 2}
$$

where $\|u\|_{\infty}:=\max _{k \in \mathbb{Z}[1, n]}\left|u_{k}\right|$.

Set

$$
\Phi(u):=\frac{u^{t} A u}{2}-\sum_{k=1}^{n} H_{k}\left(u_{k}\right),
$$

and

$$
\Psi(u):=\sum_{k=1}^{n} F_{k}\left(u_{k}\right), \quad J_{\lambda}(u):=\Phi(u)-\lambda \Psi(u),
$$

for every $u \in X$, where $H_{k}(t):=\int_{0}^{t} h_{k}(\xi) d \xi$ and $F_{k}(t):=\int_{0}^{t} f_{k}(\xi) d \xi$, for every $(k, t) \in$ $\mathbb{Z}[1, n] \times \mathbb{R}$.

Standard arguments show that $J_{\lambda} \in C^{1}(X, \mathbb{R})$, as well as that critical points of $J_{\lambda}$ are exactly the solutions of problem $\left(S_{A, \lambda}^{f, h}\right)$; see, for instance, the paper [24]. 
Lemma 1. Set

$$
L:=\max _{k \in \mathbb{Z}[1, n]} L_{k}<\lambda_{1}
$$

Then the functional $\Phi$ is coercive.

Proof. Bearing in mind (2.1), since $h_{k}$ is a Lipschitz continuous function (for every $k \in$ $\mathbb{Z}[1, n])$ with constant $L_{k} \geq 0$ and $h_{k}(0)=0$, we have

$$
\begin{aligned}
\Phi(u) & \geq \frac{\lambda_{1}}{2}\|u\|^{2}-\sum_{k=1}^{n}\left|H_{k}\left(u_{k}\right)\right| \geq \frac{1}{2}\|u\|^{2}-\sum_{k=1}^{n}\left(\int_{0}^{u_{k}}\left|h_{k}(t)\right| d t\right) \\
& \geq \frac{\lambda_{1}}{2}\|u\|^{2}-L \sum_{k=1}^{n} \int_{0}^{u_{k}}|t| d t=\frac{1}{2}\|u\|^{2}-\frac{L}{2} \sum_{k=1}^{n} u_{k}^{2} \\
& =\left(\frac{\lambda_{1}-L}{2}\right)\|u\|^{2} .
\end{aligned}
$$

Hence, by (2.5), the above relation implies that the functional $\Phi$ is coercive.

\section{MAin RESUlts}

Set

$$
A_{\infty}:=\liminf _{t \rightarrow+\infty} \frac{\sum_{k=1}^{n} \max _{|\xi| \leq t} F_{k}(\xi)}{t^{2}}, \quad \text { and } \quad B^{\infty}:=\limsup _{t \rightarrow+\infty} \frac{\sum_{k=1}^{n} F_{k}(t)}{t^{2}} .
$$

From now on we shall assume that the functions $h_{k}: \mathbb{R} \rightarrow \mathbb{R}$, for every $k \in \mathbb{Z}[1, n]$, are Lipschitz continuous with constants $L_{k}>0$ such that condition (2.5) holds.

Theorem 3. Let $A \in \mathfrak{X}_{n}$ and assume that the following inequality holds

Then, for each

$$
A_{\infty}<\frac{\lambda_{1}-L}{\operatorname{Tr}(A)+2 \sum_{i<j} a_{i j}+n L} B^{\infty} .
$$

$$
\lambda \in] \frac{\operatorname{Tr}(A)+2 \sum_{i<j} a_{i j}+n L}{2 B^{\infty}}, \frac{\lambda_{1}-L}{2 A_{\infty}}[,
$$

problem $\left(S_{A, \lambda}^{f, h}\right)$ admits an unbounded sequence of solutions.

Proof. Fix $\lambda$ as in the assertion of the theorem and put $\Phi, \Psi, J_{\lambda}$ as in (2.3) and (2.4). Since the critical points of $J_{\lambda}$ are the solutions of problem $\left(S_{A, \lambda}^{f, h}\right)$, our aim is to apply Theorem 2 part (a) to function $J_{\lambda}$. Clearly $(\Lambda)$ holds.

Therefore, our conclusion follows provided that $\gamma<+\infty$ as well as that $J_{\lambda}$ turns out to be unbounded from below. To this end, let $\left\{c_{m}\right\}$ be a real sequence such that $\lim _{m \rightarrow \infty} c_{m}=+\infty$ and

Write

$$
\lim _{m \rightarrow \infty} \frac{\sum_{k=1}^{n} \max _{|\xi| \leq c_{m}} F_{k}(\xi)}{c_{m}^{2}}=A_{\infty}
$$

$$
r_{m}:=\frac{\lambda_{1}-L}{2} c_{m}^{2}
$$


for every $m \in \mathbb{N}$.

Since, owing to (2.2), it follows that

$$
\left\{v \in X: v^{t} A v<2 r_{m}\right\} \subset\left\{v \in X:\left|v_{k}\right| \leq c_{m} \forall k \in \mathbb{Z}[1, n]\right\},
$$

and we obtain

$$
\varphi\left(r_{m}\right) \leq \frac{\sup _{v^{t} A v<2 r_{m}} \sum_{k=1}^{n} F_{k}\left(v_{k}\right)}{r_{m}} \leq \frac{\sum_{k=1}^{n} \max _{|t| \leq c_{m}} F_{k}(t)}{r_{m}}=\frac{2}{\lambda_{1}-L} \frac{\sum_{k=1}^{n} \max _{|t| \leq c_{m}} F_{k}(t)}{c_{m}^{2}} .
$$

Hence, it follows that

$$
\gamma \leq \lim _{m \rightarrow \infty} \varphi\left(r_{m}\right) \leq \frac{2}{\lambda_{1}-L} A_{\infty}<\frac{1}{\lambda}<+\infty .
$$

Now, we verify that $J_{\lambda}$ is unbounded from below. First, assume that $B^{\infty}=+\infty$. Accordingly, fix such $M$ that

$$
M>\frac{\operatorname{Tr}(A)+2 \sum_{i<j} a_{i j}+n L}{2 \lambda}
$$

and let $\left\{b_{m}\right\}$ be a sequence of positive numbers, with $\lim _{m \rightarrow \infty} b_{m}=+\infty$, such that

$$
\sum_{k=1}^{n} F_{k}\left(b_{m}\right)>M b_{m}^{2}, \quad(\forall m \in \mathbb{N}) .
$$

Thus, taking in $X$ the sequence $\left\{s_{m}\right\}$ which, for each $m \in \mathbb{N}$, is given by $\left(s_{m}\right)_{k}:=b_{m}$ for every $k \in \mathbb{Z}[1, n]$, owing to (2.1) and noting that

$$
\begin{aligned}
\Phi(u) & \leq \frac{u^{t} A u}{2}+\sum_{k=1}^{n}\left(\int_{0}^{u_{k}}\left|h_{k}(t)\right| d t\right) \\
& \leq \frac{u^{t} A u}{2}+\frac{L}{2} \sum_{k=1}^{n} u_{k}^{2} \\
& =\frac{u^{t} A u}{2}+\frac{L}{2}\|u\|^{2} .
\end{aligned}
$$

one immediately has

$$
\begin{aligned}
J_{\lambda}\left(s_{m}\right) & =\frac{s_{m}^{t} A s_{m}}{2}-\lambda \sum_{k=1}^{n} F_{k}\left(b_{m}\right) \\
& \leq \frac{\operatorname{Tr}(A)+2 \sum_{i<j} a_{i j}+n L}{2} b_{m}^{2}-\lambda \sum_{k=1}^{n} F_{k}\left(b_{m}\right) \\
& <\left(\frac{\operatorname{Tr}(A)+2 \sum_{i<j} a_{i j}+n L}{2}-\lambda M\right) b_{m}^{2} .
\end{aligned}
$$

that is, $\lim _{m \rightarrow \infty} J_{\lambda}\left(s_{m}\right)=-\infty$.

Next, assume that $B^{\infty}<+\infty$. Since

$$
\lambda>\frac{\operatorname{Tr}(A)+2 \sum_{i<j} a_{i j}+n L}{2 B^{\infty}},
$$


we can fix $\varepsilon>0$ such that

$$
\varepsilon<B^{\infty}-\frac{\operatorname{Tr}(A)+2 \sum_{i<j} a_{i j}+n L}{2 \lambda} .
$$

Therefore, also calling $\left\{b_{m}\right\}$ a sequence of positive numbers such that $\lim _{m \rightarrow \infty} b_{m}=+\infty$ and

$$
\left(B^{\infty}-\varepsilon\right) b_{m}^{2}<\sum_{k=1}^{n} F_{k}\left(b_{m}\right)<\left(B^{\infty}+\varepsilon\right) b_{m}^{2}, \quad(\forall m \in \mathbb{N})
$$

arguing as before and by choosing $\left\{s_{m}\right\}$ in $X$ as above, one has

$$
J_{\lambda}\left(s_{m}\right)<\left(\frac{\operatorname{Tr}(A)+2 \sum_{i<j} a_{i j}+n L}{2}-\lambda\left(B^{\infty}-\varepsilon\right)\right) b_{m}^{2} .
$$

So, $\lim _{m \rightarrow \infty} J_{\lambda}\left(s_{m}\right)=-\infty$.

Hence, in both cases $J_{\lambda}$ is unbounded from below. The proof is thus complete.

Remark 2. If $f_{k}$ are nonnegative continuous functions, condition $\left(\mathrm{h}_{\infty}^{L}\right)$ reads as follows

$$
\liminf _{t \rightarrow+\infty} \frac{\sum_{k=1}^{n} F_{k}(t)}{t^{2}}<\frac{\lambda_{1}-L}{\operatorname{Tr}(A)+2 \sum_{i<j} a_{i j}+n L} \limsup _{t \rightarrow+\infty} \frac{\sum_{k=1}^{n} F_{k}(t)}{t^{2}} .
$$

Arguing as in the proof of Theorem 3 and applying part (b) of Theorem 2, we obtain the following result.

Theorem 4. Let $A \in \mathfrak{X}_{n}$ and assume that the following inequality holds

$$
A_{0}<\frac{\lambda_{1}-L}{\operatorname{Tr}(A)+2 \sum_{i<j} a_{i j}+n L} B^{0} .
$$

Then, for each

$$
\lambda \in] \frac{\operatorname{Tr}(A)+2 \sum_{i<j} a_{i j}+n L}{2 B^{0}}, \frac{\lambda_{1}-L}{2 A_{0}}[,
$$

problem $\left(S_{A, \lambda}^{f}\right)$ admits a sequence of nontrivial solutions $\left\{u_{m}\right\}$ such that $\lim _{m \rightarrow \infty}\left\|u_{m}\right\|=$ $\lim _{m \rightarrow \infty}\left\|u_{m}\right\|_{\infty}=0$.

\section{Applichtion}

In this section we consider a discrete system, namely $\left(E_{\lambda}^{f, h}\right)$, given as follows

$$
\begin{gathered}
{[u(i+1, j)-2 u(i, j)+u(i-1, j)]+[u(i, j+1)-2 u(i, j)+u(i, j-1)]} \\
+\lambda f((i, j), u(i, j))+h(u(i, j))=0, \quad \forall(i, j) \in \mathbb{Z}[1, m] \times \mathbb{Z}[1, n],
\end{gathered}
$$

with boundary conditions

$$
\begin{array}{ll}
u(i, 0)=u(i, n+1)=0, & \forall i \in \mathbb{Z}[1, m], \\
u(0, j)=u(m+1, j)=0, & \forall j \in \mathbb{Z}[1, n],
\end{array}
$$

where $f: \mathbb{Z}[1, m] \times \mathbb{Z}[1, n] \times \mathbb{R} \rightarrow \mathbb{R}$ denotes a continuous function, $\lambda$ is a positive real parameter and $h: \mathbb{R} \rightarrow \mathbb{R}$ be a Lipschitz continuous function with constant $L_{h}$. 
As ambient space $X$, we consider the $m n$-dimensional Banach space $\mathbb{R}^{m n}$ endowed by the norm

$$
\|u\|:=\left(\sum_{k=1}^{m n} u_{k}^{2}\right)^{1 / 2} .
$$

Further, if $\ell \in \mathbb{N}$, the symbol $\mathfrak{M}_{\ell \times \ell}(\mathbb{R})$ stands for the linear space of all the matrices of order $\ell$ with real entries.

Let $v: \mathbb{Z}[1, m] \times \mathbb{Z}[1, n] \rightarrow \mathbb{Z}[1, m n]$ be the bijection defined by $v(i, j):=i+m(j-1)$, for every $(i, j) \in \mathbb{Z}[1, m] \times \mathbb{Z}[1, n]$.

Let us denote $w_{k}:=u\left(v^{-1}(k)\right)$ and $g_{k}\left(w_{k}\right):=f\left(v^{-1}(k), w_{k}\right)$, for every $k \in \mathbb{Z}[1, m n]$. With the above notations, problem $\left(E_{\lambda}^{f, h}\right)$ can be written as a nonlinear algebraic system of the form

$$
A w=\lambda g(w)+\widetilde{h}(w)
$$

where $A$ is given by

$$
A:=\left(\begin{array}{ccccccccc}
D & -I_{m} & 0 & 0 & \cdots & 0 & 0 & 0 & 0 \\
-I_{m} & D & -I_{m} & 0 & \cdots & 0 & 0 & 0 & 0 \\
0 & -I_{m} & D & -I_{m} & \cdots & 0 & 0 & 0 & 0 \\
0 & 0 & -I_{m} & D & \cdots & 0 & 0 & 0 & 0 \\
& & & & \ddots & & & & \\
0 & 0 & 0 & 0 & \cdots & D & -I_{m} & 0 & 0 \\
0 & 0 & 0 & 0 & \cdots & -I_{m} & D & -I_{m} & 0 \\
0 & 0 & 0 & 0 & \cdots & 0 & -I_{m} & D & -I_{m} \\
0 & 0 & 0 & 0 & \cdots & 0 & 0 & -I_{m} & D
\end{array}\right) \in \mathfrak{M}_{m n \times m n}(\mathbb{R})
$$

in which $D$ is defined by

$$
D:=\left(\begin{array}{ccccccccc}
4 & -1 & 0 & 0 & \cdots & 0 & 0 & 0 & 0 \\
-1 & 4 & -1 & 0 & \cdots & 0 & 0 & 0 & 0 \\
0 & -1 & 4 & -1 & \cdots & 0 & 0 & 0 & 0 \\
0 & 0 & -1 & 4 & \cdots & 0 & 0 & 0 & 0 \\
& & & & \ddots & & & & \\
0 & 0 & 0 & 0 & \cdots & 4 & -1 & 0 & 0 \\
0 & 0 & 0 & 0 & \cdots & -1 & 4 & -1 & 0 \\
0 & 0 & 0 & 0 & \cdots & 0 & -1 & 4 & -1 \\
0 & 0 & 0 & 0 & \cdots & 0 & 0 & -1 & 4
\end{array}\right) \in \mathfrak{M}_{m \times m}(\mathbb{R})
$$

$I_{m} \in \mathfrak{M}_{m \times m}(\mathbb{R})$ is the identity matrix and $g(w):=\left(g_{1}\left(w_{1}\right), \ldots, g_{m n}\left(w_{m n}\right)\right)^{t}, \tilde{h}(w):=$ $\left(h\left(w_{1}\right), \ldots, h\left(w_{m n}\right)\right)^{t}$, for every $w \in X$.

In [9], Ji and Yang studied the structure of the spectrum of the above (non-perturbed) Dirichlet problem. By their result we have that $A \in \mathfrak{X}_{m n}$.

It is easy to observe that the solutions of $\left(E_{\lambda}^{f, h}\right)$ are the critical points of the $C^{1}$-functional

$$
J_{\lambda}(w):=\frac{w^{t} A w}{2}-\lambda \sum_{k=1}^{m n} \int_{0}^{w_{k}} g_{k}(t) d t-\sum_{k=1}^{m n} \int_{0}^{w_{k}} h(t) d t, \quad \forall w \in X .
$$

Denote by $\lambda_{A}$ the first eigenvalue of the matrix $A$. By using the above variational framework, Theorem 3 assumes the following form. 
Theorem 5. Assume that $\lambda_{A}<L_{h}$, in addition to

$$
\left(\mathrm{h}_{\infty}^{h}\right) \quad \liminf _{t \rightarrow+\infty} \frac{\sum_{k=1}^{m n} \max _{|\xi| \leq t} \int_{0}^{\xi} g_{k}(s) d s}{t^{2}}<\frac{\lambda_{A}-L_{h}}{\left(2+L_{h}\right)(m+n)} \limsup _{t \rightarrow+\infty} \frac{\sum_{k=1}^{m n} \int_{0}^{t} g_{k}(s) d s}{t^{2}} .
$$

Then for each

$$
\lambda \in] \frac{\left(2+L_{h}\right)(m+n)}{2 B^{\infty}}, \frac{\lambda_{A}-L_{h}}{2 A_{\infty}}[,
$$

problem $\left(E_{\lambda}^{f, h}\right)$ admits an unbounded sequence of solutions.

Remark 3. Substituting $\xi \rightarrow+\infty$ with $\xi \rightarrow 0^{+}$in Theorem 5 , the same statement as Theorem 4 is easily proved.

Remark 4. We just point out that Theorem 1 in Introduction directly follows by Theorem 5 assuming that $L_{h}<\lambda_{A}$.

In conclusion we present here a direct consequence of Theorem 5 .

Example 1. Let $h: \mathbb{R} \rightarrow \mathbb{R}$ be a Lipschitz continuous function with constant $L_{h}<\lambda_{A}$ and let

for every $n \in \mathbb{N}$.

$$
a_{n}:=\frac{2 n !(n+2) !-1}{4(n+1) !}, \quad b_{n}:=\frac{2 n !(n+2) !+1}{4(n+1) !},
$$

Let $\left\{g_{n}\right\}$ be a sequence of non-negative functions given by

$$
g_{n}(\xi):=\sqrt{\frac{1}{16(n+1) !^{2}}-\left(\xi-\frac{n !(n+2)}{2}\right)^{2}}, \quad \forall n \in \mathbb{N} .
$$

and define $f: \mathbb{R} \rightarrow \mathbb{R}$ as follows

$$
f(\xi):= \begin{cases}{\left[(n+1) !^{2}-n !^{2}\right] \frac{g_{n}(\xi)}{\int_{a_{n}}^{b_{n}} g_{n}(t) d t}} & \text { if } \xi \in \bigcup_{n=1}^{\infty}\left[a_{n}, b_{n}\right] \\ 0 & \text { otherwise. }\end{cases}
$$

One has

$$
\int_{n !}^{(n+1) !} f(t) d t=\int_{a_{n}}^{b_{n}} f(t) d t=(n+1) !^{2}-n !^{2}
$$

and

$$
F\left(a_{n}\right)=n !^{2}-1, \quad F\left(b_{n}\right)=(n+1) !^{2}-1
$$

for every $n \in \mathbb{N}$.

Hence

$$
\lim _{n \rightarrow+\infty} \frac{F\left(b_{n}\right)}{b_{n}^{2}}=4, \quad \lim _{n \rightarrow+\infty} \frac{F\left(a_{n}\right)}{a_{n}^{2}}=0 .
$$

Therefore, we can prove that $\liminf _{\xi \rightarrow+\infty} \frac{F(\xi)}{\xi^{2}}=0$ and $\limsup _{\xi \rightarrow+\infty} \frac{F(\xi)}{\xi^{2}}=4$.

Then, for every

$$
\lambda>\frac{\left(2+L_{h}\right)(m+n)}{8 m n},
$$


the following problem

$$
\begin{gathered}
{[u(i+1, j)-2 u(i, j)+u(i-1, j)]+[u(i, j+1)-2 u(i, j)+u(i, j-1)]} \\
+\lambda f(u(i, j))+h(u(i, j))=0, \quad \forall(i, j) \in \mathbb{Z}[1, m] \times \mathbb{Z}[1, n]
\end{gathered}
$$

with boundary conditions

$$
\begin{array}{ll}
u(i, 0)=u(i, n+1)=0, & \forall i \in \mathbb{Z}[1, m], \\
u(0, j)=u(m+1, j)=0, & \forall j \in \mathbb{Z}[1, n],
\end{array}
$$

admits an unbounded sequence of solutions.

Remark 5. We refer to the paper of Galewski and Orpel [5] for some multiplicity results on discrete partial difference equations as well as to the monograph of Cheng [4] for their discrete geometrical interpretation. See also the papers $[14,16,17,18,19]$ for recent contributions on discrete problems.

\section{REFERENCES}

[1] R. P. Agarwal, Difference Equations and Inequalities: theory, methods and applications, Marcel Dekker, New York-Basel, 2000.

[2] C. Bereanu and J. Mawhin, Existence and multiplicity results for nonlinear second order difference equations with Dirichlet boundary conditions, Math. Bohem. 131 (2006), 145-160.

[3] X. Cai and J. Yu, Existence theorems of periodic solutions for second-order nonlinear difference equations, Adv. Difference Equ. 2008 (2008) Article ID 247071.

[4] S.S. Cheng, Partial difference equations, Taylor \& Francis, London (2003).

[5] M. Galewski and A. Orpel, On the existence of solutions for discrete elliptic boundary value problems, Appl. Anal. 89 (2010), 1879-1891.

[6] M. Galewski and J. Smejda, On variational methods for nonlinear difference equations, J. Comput. Appl. Math. 233: 11 (2010), 2985-2993.

[7] T. He and Y. Su, On discrete fourth-order boundary value problems with three parameters, J. Comput. Appl. Math. 233 (2010), 2506-2520.

[8] J. Henderson and H.B. Thompson, Existence of multiple solutions for second order discrete boundary value problems, Comput. Math. Appl. 43 (2002), 1239-1248.

[9] J. Ji and B. Yang, Eigenvalue comparisons for boundary value problems of the discrete elliptic equation, Commun. Appl. Anal. 12 (2) (2008), 189-197.

[10] W. G. Kelly and A. C. Peterson, Difference Equations, An introduction with applications, Academic Press, San Diego-NewYork, 1991.

[11] A. Kristály, V. Rădulescu and Cs. Varga, Variational Principles in Mathematical Physics, Geometry, and Economics: Qualitative Analysis of Nonlinear Equations and Unilateral Problems, Encyclopedia of Mathematics and its Applications, No. 136, Cambridge University Press, Cambridge, 2010.

[12] A. Kristály, M. Mihăilescu, V. Rădulescu and S. Tersian, Spectral estimates for a nonhomogeneous difference problem, Commun. Contemp. Math. 12 (2010), n. 6, 1015-1029.

[13] A. Kristály, M. Mihăilescu and V. Rădulescu, Discrete boundary value problems involving oscillatory nonlinearities: small and large solutions, J. Difference Equ. Appl. 17 (2011), 1431-1440.

[14] N. Marcu and G. Molica Bisci, Existence and multiplicity results for nonlinear discrete inclusions, Electron. J. Differential Equations, 2012, 1-13.

[15] M. Mihăilescu, V. Rădulescu and S. Tersian, Eigenvalue Problems for Anisotropic Discrete Boundary Value Problems, J. Difference Equ. Appl. 15 (2009), 557-567.

[16] G. Molica Bisci and D. Repovš, On some variational algebraic problems, Adv. Nonlinear Analysis 2 (2013), 127-146.

[17] G. Molica Bisci and D. Repovš, Nonlinear Algebraic Systems with discontinuous terms, J. Math. Anal. Appl. 398 (2013), 846-856.

[18] G. Molica Bisci and D. Repovš, On sequences of solutions for discrete anisotropic equations, Expo. Math. 32 (2014), no. 3, 284-295. 
[19] G. Molica Bisci and D. Repovš, Existence of solutions for p-Laplacian discrete equations, Appl. Math. Comput. 242 (2014), 454-461.

[20] D. Motreanu and V. Rădulescu, Variational and non-variational methods in nonlinear analysis and boundary value problems, Nonconvex Optimization and its Applications, 67, Kluwer Academic Publishers, Dordrecht, 2003.

[21] B. Ricceri, A general variational principle and some of its applications, J. Comput. Appl. Math. 133 (2000), 401-410.

[22] P. Stehlík, On variational methods for periodic discrete problems, J. Difference Equ. Appl. 14 (3) (2008), 259-273.

[23] Y. Yang and J. Zhang, Existence results for a nonlinear system with a parameter, J. Math. Anal. Appl. 340 (2008), n. 1, 658-668.

[24] Y. Yang and J. Zhang, Existence and multiple solutions for a nonlinear system with a parameter, Nonlinear Anal. 70 (2009), n. 7, 2542-2548.

[25] G. Zhang, Existence of non-zero solutions for a nonlinear system with a parameter, Nonlinear Anal. 66 (6) (2007), 1410-1416.

[26] G. Zhang and L. Bai, Existence of solutions for a nonlinear algebraic system, Discrete Dyn. Nat. Soc. (2009), 1-28.

[27] G. Zhang and S.S. Cheng, Existence of solutions for a nonlinear algebraic system with a parameter, J. Math. Anal. Appl. 314 (2006), 311-319.

[28] G. Zhang and W. Feng, On the number of positive solutions of a nonlinear algebraic system, Linear Algebra Appl. 422 (2007), 404-421.

Dipartimento P.A.U., Università degli Studi Mediterranea di Reggio Calabria, Salita MelisSARi - Feo di Vito, 89124 Reggio Calabria, Italy

E-mail address: gmolica@unirc.it

Faculty of Education, and Faculty of Mathematics and Physics, University of Ljubluana, POB 2964, Luubluana, Slovenia

E-mail address: dusan.repovs@guest.arnes.si 